\title{
Role of lincRNA-p21 in the protective effect of macrophage inhibition factor against hypoxia/serum deprivation-induced apoptosis in mesenchymal stem cells
}

\author{
WENZHENG XIA ${ }^{1^{*}}$, LEI ZHUANG ${ }^{2 *}$ and MENG HOU ${ }^{3}$ \\ Departments of ${ }^{1}$ Neurosurgery, ${ }^{2}$ Hepatobiliary Surgery and ${ }^{3}$ Radiation Oncology, \\ First Affiliated Hospital, Wenzhou Medical University, Wenzhou, Zhejiang 325000, P.R. China
}

Received January 16, 2018; Accepted June 28, 2018

DOI: $10.3892 /$ ijmm.2018.3767

\begin{abstract}
Stem cell transplantation is a promising clinical strategy for curing ischemic cardiomyopathy. However, its efficacy is impaired by low cell survival following transplantation, partly caused by insufficient resistance of the transplanted stem cells to severe oxidative stress at the injury site. In the current study, it was demonstrated that the small-molecule macrophage migration inhibitory factor (MIF) enhanced the defense of bone marrow-derived mesenchymal stem cells (MSCs) against hypoxia/serum deprivation (SD)-induced apoptosis in vitro. MIF significantly suppressed apoptosis and caspase family activities through inhibition of long intergenic noncoding (linc) RNA-p21 to maintain activation of the Wnt/ $\beta$-catenin signaling pathway. The regulatory loop between MIF and the lincRNA-p21-Wnt/ $\beta$-catenin signaling pathway was identified to be associated with the inhibition of oxidative stress. The involvement of the lincRNA-p21-Wnt/ $\beta$-catenin signaling pathway in the effects of MIF in MSCs by overexpression of lincRNA-p21and silencing $\beta$-catenin using small interfering RNA was also demonstrated, both of which abolished the anti-apoptotic and anti-oxidative effects of MIF in MSCs under hypoxia/SD conditions. In conclusion, MIF protected MSCs from hypoxia/SD-induced apoptosis by interacting with lincRNA-p21, leading to activation of the downstream $\mathrm{Wnt} / \beta$-catenin signaling pathway and decreased oxidative stress. Thus, treatment with MIF may have important therapeutic implications in improving MSC survival and therapeutic efficiency.
\end{abstract}

Correspondence to: Dr Meng Hou, Department of Radiation Oncology, First Affiliated Hospital, Wenzhou Medical University, 2 Fuxue Lane, Wenzhou, Zhejiang 325000, P.R. China

E-mail: 244517813@qq.com

${ }^{*}$ Contributed equally

Key words: mesenchymal stem cell, hypoxia/serum deprivation, apoptosis, lincRNA-p21, Wnt/ $\beta$-catenin signaling pathway, oxidative stress

\section{Introduction}

Ischemic cardiomyopathy arising from myocardial ischemia is the leading cause of morbidity and mortality worldwide (1). There are numerous therapeutic options for ischemic cardiomyopathy, including the potential use of mesenchymal stem cells (MSCs) for tissue repair, as demonstrated in clinical trials for intractable diseases $(2,3)$. However, the therapeutic efficacy of MSCs has been hindered by the low survival rate of transplanted cells. The survival and retention of MSCs following transplantation are adversely affected by the harmful ischemic microenvironment (4), and myocardial oxidative stress restricts the therapeutic effects of MSCs on cardiac repair (5). It is therefore important to develop novel strategies to promote donor cell survival to improve the efficacy of stem-cell-based therapy for ischemic cardiomyopathy.

Macrophage migration inhibitory factor (MIF) is a widely expressed pleiotropic cytokine and is considered to be an important therapeutic target for treating cardiovascular disease (6). MIF regulates cellular activities through transcriptional regulation of inflammatory gene products, modulation of cell proliferation, cell cycle control and metabolism, and by inhibition of apoptosis (7). MIF protects against myocardial ischemia/reperfusion injury primarily through preventing redox stress (8). MIF is a good anti-apoptotic factor candidate, and in an ischemia/reperfusion injury model, MIF was also demonstrated to protect against oxidative stress-mediated cardiomyocyte apoptosis (9). In the current study, the protective effects of exogenous MIF were determined in MSCs exposed to hypoxia/serum deprivation (SD) to mimic the ischemic environment.

Long non-coding RNAs (lncRNAs) are RNA transcripts $>200$ bp in length with no apparent protein-coding ability (10). Increasing evidence suggests that lncRNAs affect numerous cellular functions, and participate in diverse physiological and pathological processes (11), including development, differentiation, stem cell pluripotency and apoptosis (12). Long intergenic noncoding RNA-p21 (lincRNA-p21) is a p53-dependent transcriptional target gene involved in proliferation, the cell cycle, metabolism and apoptosis (13). Previous studies have demonstrated that lincRNA-p21 is associated with oxidative-stress-induced apoptosis $(14,15)$, and participates in 
senescence-induced cellular injury through the induction of oxidative stress (16). MIF inhibited the p21-dependent death signaling in keratinocytes (17). We therefore hypothesized that lincRNA-p21 may be a target gene inhibited by MIF, subsequently preventing hypoxia/SD-induced injury.

Wnt/ $\beta$-catenin signaling is known to serve essential roles in cell growth, survival and apoptosis (18). As a target gene of lincRNA-p21, $\beta$-catenin is associated with cellular apoptosis, proliferation and oxidative stress (19). lincRNA-p21 was reported to inhibit hepatic stellate cell proliferation through inactivation of the Wnt/ $\beta$-catenin signaling pathway (20). Furthermore, lincRNA-p21negatively regulates $\beta$-catenin translation at the post-transcriptional level and contributes to glioma stem cell apoptosis (15). In addition, inhibition of Wnt/ $\beta$-catenin signaling induced by lincRNA-p21 contributes to cellular senescence in MSCs by inhibiting cellular oxidative stress (16). However, the role of lincRNA-p21-associated inhibition of the Wnt/ $\beta$-catenin pathway in hypoxia/SD-induced MSC apoptosis remains unclear.

We proposed that exogenous MIF prevents hypoxia/SDinduced apoptosis and inhibits oxidative stress. Thus, the effect of MIF on hypoxia/SD-induced apoptosis of MSCs and its associated signaling pathways were examined in the present study.

\section{Materials and methods}

Reagents. Dulbecco's modified Eagle's medium (DMEM) and fetal bovine serum (FBS) were purchased from GE Healthcare Life Sciences (HyClone; Logan, UT, USA), TRIzol ${ }^{\circledR}$ reagent was obtained from Thermo Fisher Scientific, Inc. (Invitrogen; Waltham, MA, USA), and the Transcriptor First Strand cDNA Synthesis kit, FastStart Universal SYBR ${ }^{\circledR}$-Green Master (Rox) and X-tremeGENE HP DNA transfection reagent were purchased from Roche Diagnostics (Basel, Switzerland). The Annexin V-fluorescein isothiocyanate (FITC) Apoptosis Detection kit was obtained from BD Biosciences (BD Pharmingen; Franklin Lakes, NJ, USA). Rabbit monoclonal antibodies against $\beta$-catenin (\#8480; $1: 1,000)$ and $\beta$-actin (\#4970; 1:1,000) were obtained from Cell Signaling Technology, Inc. (Danvers, MA, USA) and horseradish peroxidase-conjugated anti-rabbit secondary antibodies (\#7074; 1:2,000) were from Santa Cruz Biotechnology, Inc. (Dallas, TX, USA). Small interfering RNAs (siRNAs) targeting lincRNA-p21 and $\beta$-catenin transcripts were purchased from Thermo Fisher Scientific, Inc. The ELISA kit for MIF was purchased from Abcam (\#ab7207; Cambridge, UK), and the Mitochondrial Membrane Potential assay kit with JC-1 (\#C2006) and Reactive Oxygen Species (ROS) assay kit (\#S0033) were purchased from Beyotime Institute of Biotechnology (Jiangsu, China). The Superoxide Dismutase (SOD) Activity Colorimetric assay (\#ab211096), Lipid Peroxidation (malondialdehyde; MDA) assay kits (\#ab118970) and mouse recombinant MIF were purchased from Abcam.

Cell culture and treatment. Bone marrow-derived mesenchymal stem cells (MSCs) were isolated using a standard protocol, as described previously (16). A total of 12 male mice (mean age, 6 months; mean weight, 22.85 $\pm 2.62 \mathrm{~g}$ ) were purchased from the Laboratory Animal Center of Wenzhou
Medical University (Wenzhou, China). Mice were kept to a $12 \mathrm{~h} \mathrm{light} / 12 \mathrm{~h}$ dark cycle at $21 \pm 2^{\circ} \mathrm{C}$ with $30-70 \%$ relative humidity. Food and water was freely available throughout. All animal procedures were approved by the Wenzhou Medical University Institutional Animal Care and Use Committee (Wenzhou, China). Briefly, bone marrow was isolated from mouse femurs and tibias by flushing with PBS. Adherent MSCs were cultured at $37^{\circ} \mathrm{C}$ and $5 \% \mathrm{CO}_{2}$ in high-glucose DMEM supplemented with $10 \%$ FBS and $1 \%$ penicillin/streptomycin. Third-passage MSCs were used for experiments.

Apoptosis was induced in vitro by hypoxia and SD to mimic the in vivo conditions of the ischemic myocardium, as previously reported (21). Apoptosis was induced by incubating MSCs in serum-free DMEM in a glove box (model no. 855-AC; Plas Labs, Inc., Lansing, MI, USA) with a regulated atmosphere (anaerobic chamber) to scavenge free oxygen (hypoxia/SD group). For MIF treatment, cells were cultured with DMEM containing $100 \mathrm{ng} / \mathrm{ml}$ recombinant MIF and incubated at $37^{\circ} \mathrm{C}$ for various periods of time, as reported previously (22). Untreated cells were used as the control group throughout.

Flow cytometric analysis of cell apoptosis. The effects of MIF on apoptosis were determined by detecting phosphatidylserine exposure on cell plasma membranes using an Annexin V-FITC Apoptosis Detection kit, according to the manufacturer's protocol. Briefly, cells were harvested $\left(4^{\circ} \mathrm{C}, 5 \mathrm{~min}, 12,000 \mathrm{x} \mathrm{g}\right)$ and washed in ice-cold PBS, resuspended in $300 \mu$ l binding buffer, and incubated with $5 \mu$ l Annexin V-FITC solution for $30 \mathrm{~min}$ at $4^{\circ} \mathrm{C}$ in the dark, followed by further incubation in $5 \mu \mathrm{l}$ propidium iodide for $5 \mathrm{~min}$ at $4^{\circ} \mathrm{C}$. The cells were then analyzed immediately using bivariate flow cytometry with a BD FACSCanto II equipped with BD FACSDiva software (version 8.0.1; BD Biosciences).

Calculation of caspase 3/7/8 activities. Caspase 3/7/8 activities in MSCs were determined as described previously (23). Briefly, activities of caspases 3/7/8 in cell lysates of MSCs, MSCs under hypoxia/SD conditions and MSCs treated with MIF under hypoxia/SD conditions were measured using a Cell Meter Caspase 3/7/8 Activity Apoptosis assay kit (AAT Bioquest, Sunnyvale, CA, USA) according to the manufacturer's protocol. Results were read at $520 \mathrm{~nm}$ with a microplate reader and expressed as the fold change in caspase $3 / 7 / 8$ activity compared with the control.

Reverse transcription-quantitative polymerase chain reaction $(R T-q P C R)$. Expression levels of several genes were analyzed using RT-qPCR. Briefly, total cellular RNA was isolated using TRIzol reagent and reverse transcribed using a Transcriptor First Strand cDNA Synthesis kit according to the manufacturer's protocol. qPCR was performed using the Fast Start Universal SYBR Master and the Applied Biosystems Step One Plus Real-Time PCR system (Applied Biosystems; Thermo Fisher Scientific, Inc.). The thermocycling conditions were as follows: $95^{\circ} \mathrm{C}$ for $10 \mathrm{~min}$, followed by 40 cycles of $15 \mathrm{sec}$ at $95^{\circ} \mathrm{C}$ and $1 \mathrm{~min}$ at $60^{\circ} \mathrm{C}$. The threshold number of cycles (Cq) was set within the exponential phase of the PCR. The $\Delta \mathrm{Cq}$ value for each target gene was calculated by subtracting the $\mathrm{Cq}$ value for GAPDH (internal control) from that of the 
Table I. Primer sequences.

\begin{tabular}{ll}
\hline Genes & \multicolumn{1}{c}{ Sequences } \\
\hline lincRNA-p21 & \\
F & 5'-CCT GTC CAC TCG CTT TC-3' \\
R & 5'-GGA ACT GGA GAC GGA ATG TC-3' \\
$\beta$-catenin & \\
F & 5'-TAG TGT GAC AAG CTG AGT ATG CGA-3' \\
R & 5'-CTG GAG CGT CTG ATG AG-3' \\
GAPDH & \\
F & \\
R & 5'-GGA GCC AAA AGG GTC ATC AT-3' \\
siRNA-LincRNA-p21 & 5'-GTG ATG GCA TGG ACT GTG GT-3' \\
siRNA- $\beta$-catenin & UGA AAA GAG CCG UGA GCU A \\
siRNA-NT & CTC ACT TGC AAT AAT TAC AAA \\
\hline
\end{tabular}

siRNA, small interfering RNA; linc, long intergenic noncoding; NT, non-targeting; F, forward; R, reverse.

target gene. Relative gene expression levels were calculated by comparing the $\Delta \mathrm{Cq}$ values between the control and experimental conditions for each target PCR using the following

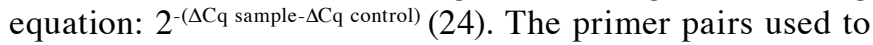
detect the mRNA levels of target genes are listed in Table I.

Western blot analysis. MSCs were lysed with ice-cold lysis buffer (Beyotime Institute of Biotechnology) to obtain total protein, then $\beta$-catenin and $\beta$-actin expression levels were evaluated using western blotting. Cellular extracts were prepared according to the manufacturer's protocol. Protein samples were quantified and separated by SDS-PAGE. Western blotting was performed as described previously (25). Quantity One software (version 4.5.2; Bio-Rad Laboratories, Inc., Hercules, CA, USA) was used for densitometric analysis.

lincRNA-p21 and $\beta$-catenin siRNA knockdown. MSCs were transfected using X-treme GENE HP DNA Transfection reagent according to the manufacturer's protocol. Briefly, MSCs ( $1 \times 10^{5}$ cells/well) were cultured in 6-well plates for $24 \mathrm{~h}$ and then treated with the transfection reagent (siRNA weight ratio of 3:1) for $20 \mathrm{~min}$. This was followed by the addition of a mixture containing $100 \mathrm{nM}$ siRNA and incubation in $2 \mathrm{ml}$ DMEM for $48 \mathrm{~h}$ at $37^{\circ} \mathrm{C}$. Scrambled non-targeting siRNA (siRNA-NT) was used as a negative control. The knockdown efficiency was determined by RT-qPCR, as aforementioned. The sequences are listed in Table I.

Plasmid transfection. Adenoviral vectors expressing lincRNA-p21 (Ad-lincRNA-p21 group) and control scrambled sequence (Ad-ctrl group) were designed and synthesized by Shanghai GeneChem Co., Ltd. (Shanghai, China). MSCs were transfected using Lipofectamine ${ }^{\mathrm{TM}} 2000$ (Invitrogen; Thermo Fisher Scientific, Inc.) at a final concentration of $100 \mathrm{nM}$.

Evolution of mitochondrial transmembrane potential. Cells were cultured in complete DMEM in 96-well microtiter plates at $37^{\circ} \mathrm{C}$ for 1 day to achieve $1 \times 10^{5}$ cells/well. The cells were then washed with PBS and incubated with $5 \mu \mathrm{g} / \mathrm{ml} \mathrm{JC}-1$ at $37^{\circ} \mathrm{C}$ for $15 \mathrm{~min}$. Following two washes with PBS, time-dependent JC-1 fluorescence was recorded using an ELISA plate reader. The fluorescent probe was excited at $490 \mathrm{~nm}$ and the emission was read alternately at 530 and $590 \mathrm{~nm}$.

ROS measurement. Levels of intracellular ROS were determined using 2,7-dichlorodihydrofluorescein diacetate (Beyotime Institute of Biotechnology), following the manufacturer's protocol. The fluorescence intensity of the cells was measured using a fluorescence spectrophotometer, with excitation and emission wavelengths of 488 and $525 \mathrm{~nm}$, respectively.

SOD activity. SOD activity in MSCs was determined using a SOD Activity Colorimetric assay according to the manufacturer's protocol. Briefly, protein was isolated from MSCs using lysis buffer, and SOD activity was measured in $10 \mu \mathrm{g}$ of total protein extract. Absorbance was measured at $450 \mathrm{~nm}$.

Lipid peroxidation assays. Lipid peroxidation was monitored using an assay kit to measure the formation of MDA, according to the manufacturer's protocol. Briefly, MSCs $\left(1 \times 10^{6}\right.$ cells) were homogenized on ice in $300 \mu 1$ of MDA lysis buffer (with $3 \mu \mathrm{l}$ of $100 \mathrm{X}$ butylated hydroxytoluene), and then centrifuged $\left(4^{\circ} \mathrm{C}, 13,000 \mathrm{x} \mathrm{g}, 10 \mathrm{~min}\right)$ to remove insoluble material. The supernatant $(200 \mu \mathrm{l})$ was added to $600 \mu \mathrm{l}$ of thiobarbituric acid and incubated at $95^{\circ} \mathrm{C}$ for $60 \mathrm{~min}$. The samples were cooled to room temperature in an ice bath for $10 \mathrm{~min}$, and the absorbance at $532 \mathrm{~nm}$ was measured spectrophotometrically.

Statistical analysis. Data are expressed as the mean \pm standard deviation following three repeats. Differences among groups were analyzed using one-way analysis of variance followed by Tukey's multiple comparisons test, and comparisons between two groups were evaluated by Student's t-tests using SPSS software (version 19.0; IBM, Corp., Armonk, NY, USA). P<0.05 was considered to indicate a statistically significant difference. 

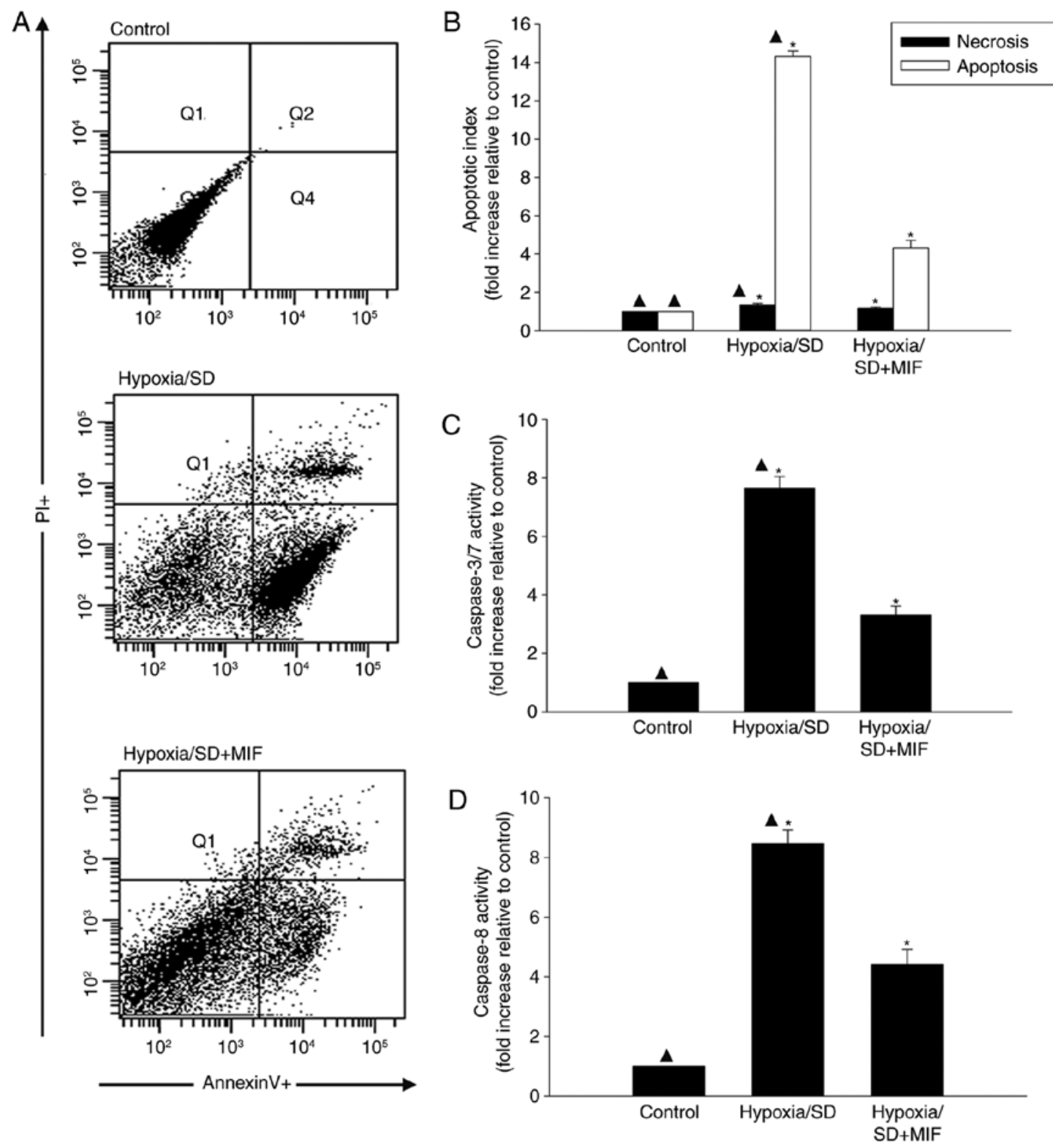

Figure 1. MIF ameliorated cell injury induced by hypoxia/SD in MSCs. MIF $(100 \mathrm{ng} / \mathrm{ml})$ was maintained in the incubation medium throughout the hypoxia/SD treatment period. (A) Representative flow cytometric dot plots of apoptotic cells following Annexin V/propidium iodide staining and (B) quantification of apoptosis. (C) Activities of caspases 3/7 and (D) caspase 8 in cell lysates measured using ELISA. Each column represents the mean \pm standard deviation of three independent experiments; ${ }^{*} \mathrm{P}<0.05$ vs. control; ${ }^{\wedge} \mathrm{P}<0.05$ vs. hypoxia/SD+MIF. MIF, macrophage migration inhibitory factor; MSC, mesenchymal stem cells; SD, serum deprivation.

\section{Results}

MIF ameliorates cell injury induced by hypoxia/SD in MSCs. Hypoxia/SD induced MSC injury, with maximal injury at $24 \mathrm{~h}$. To determine if MIF protects MSCs from hypoxia/SD-induced injury, MSCs were exposed to MIF (100 $\mathrm{ng} / \mathrm{ml})$ followed by hypoxia/SD for $24 \mathrm{~h}$ and apoptosis rates were determined by flow cytometry. MIF demonstrated a significant anti-apoptotic effect of the hypoxia/SD model, as demonstrated by Annexin V-FITCFACS analysis (Fig. 1A and B).

The anti-apoptotic effects of MIF were further examined by measuring the effects of MIF pretreatment on changes in caspases 3/7 and caspase 8 following hypoxia/SD induction. MIF pretreatment significantly reduced the stress-induced increases in caspase activities (Fig. 1C and D).
MIF protects MSCs from hypoxia/SD-induced apoptosis by inhibiting the expression of lincRNA-p21. lincRNA-p21 has been reported to be associated with cellular apoptosis (26). To determine if lincRNA-p21 was involved in hypoxia/SD-induced apoptosis, its expression was evaluated in MSCs exposed to hypoxia/SD for $24 \mathrm{~h}$. lincRNA-p21 was significantly increased in MSCs following hypoxia/SD treatment, as demonstrated by RT-qPCR analysis (Fig. 2A). Furthermore, the hypoxia/SD-induced increase was significantly attenuated by exogenous MIF treatment (Fig. 2A).

The role of MIF-induced inhibition of lincRNA-p21in protecting MSCs from hypoxia/SD-induced apoptosis was further examined. MIF was added to MSCs prior to hypoxia/SD treatment and apoptosis was measured by flow cytometry. In a parallel experiment, MSCs were transfected 

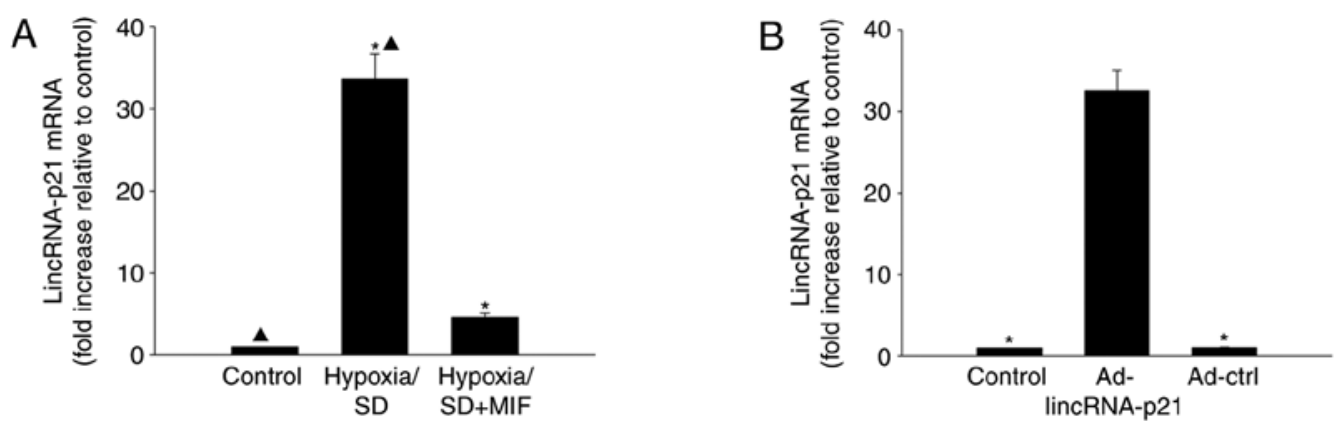

C
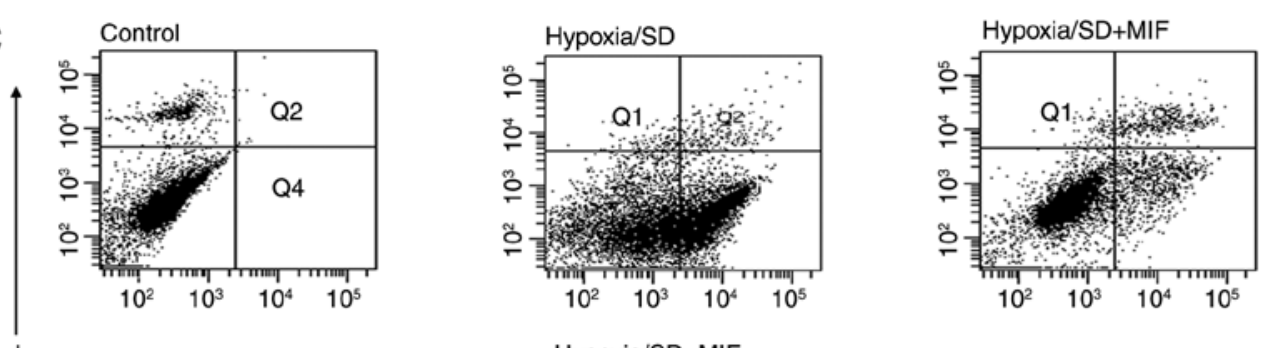

$\frac{ \pm}{0}$

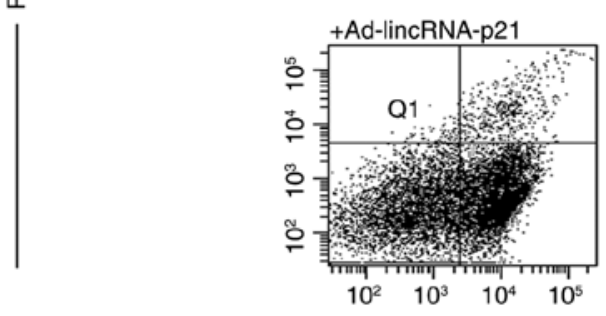

Hypoxia/SD+MIF

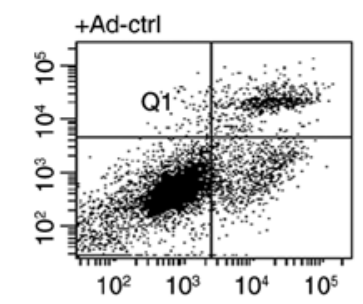
AnnexinV+
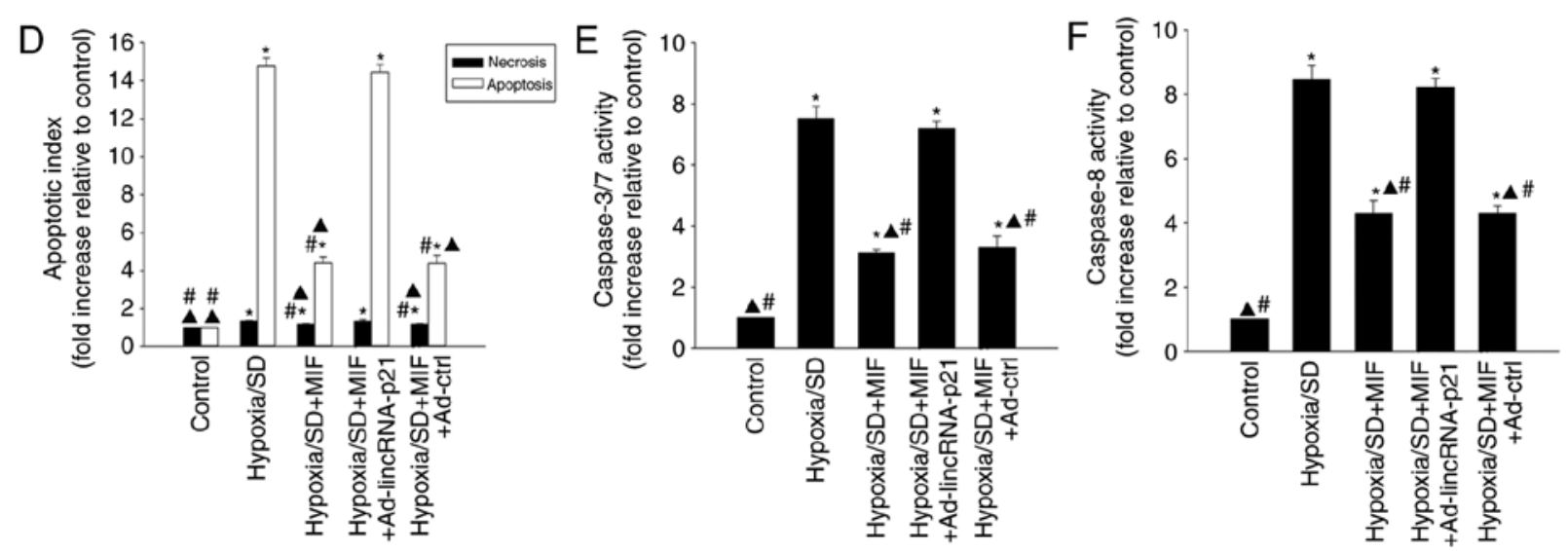

Figure 2. MIF protected MSCs against hypoxia/SD-induced apoptosis by inhibiting expression of lincRNA-p21. (A) LincRNA-p21 mRNA levels in MSCs cultured under hypoxia/SD conditions with or without MIF were analyzed by RT-qPCR; "P<0.05 vs. control; ${ }^{\mathbf{}} \mathrm{P}<0.05$ vs. hypoxia/SD+MIF. (B) MSCs were transfected with Ad-lincRNA-p21 or Ad-ctrl. The transfection efficiency was demonstrated by RT-qPCR; ${ }^{*} \mathrm{P}<0.05$ vs. Ad-lincRNA-p21. MSCs were then subjected to hypoxia/SD for $24 \mathrm{~h}$. In parallel experiments, cells were treated with MIF (100 ng/ml) prior to hypoxia/SD, and MIF was maintained in the incubation medium throughout the hypoxia/SD treatment period. (C) Representative flow cytometric dot plots of apoptotic cells following Annexin V/propidium iodide staining and (D) quantification of apoptosis. (E) Activities of caspases 3/7 and (F) caspase 8 in cell lysates were measured by ELISA. Each column represents the mean \pm standard deviation of three independent experiments; ${ }^{*} \mathrm{P}<0.05$ vs. control; ${ }^{\mathbf{4}} \mathrm{P}<0.05$ vs. hypoxia/SD; ${ }^{*} \mathrm{P}<0.05$ vs. hypoxia/SD+MIF+Ad-lincRNA-p21. MIF, macrophage migration inhibitory factor; MSC, mesenchymal stem cells; SD, serum deprivation; linc, long intergenic noncoding; RT-qPCR, reverse transcription-quantitative polymerase chain reaction; Ad-lincRNA-p21, adenoviral vectors expressing lincRNA-p21; Ad-ctrl, adenoviral vectors expressing control scrambled sequence.

with Ad-lincRNA-p21 prior to treatment with MIF (Fig. 2B) and cultured under hypoxia/SD conditions. MIF treatment significantly decreased cellular apoptosis (Fig. 2C and D), and reduced caspases $3 / 7$ and caspase 8 activities compared with cells under hypoxia/SD treatment alone (Fig. 2E and F). In addition, these effects were significantly abolished by lincRNA-p21 overexpression (Fig. 2C-F).
MIF restores the lincRNA-p21-Wnt/ $\beta$-catenin signaling pathway altered by hypoxia/SD induction. The Wnt/ $\beta$-catenin signaling pathway is a known target of lincRNA-p21, and has been reported to be involved in cellular injury in MSCs (16). In the present study, $\beta$-catenin protein expression levels in MSCs were significantly decreased by hypoxia/SD compared with control cells, which was restored following 
pre-treatment with MIF. Overexpression of lincRNA-p21by Ad-lincRNA-p21 transfection abolished the effect of MIF, while transfection with the control vector had no significant effect (Fig. 3A and B). The mechanism underlying the modulation of lincRNA-p21-Wnt/ $\beta$-catenin signaling by MIF in hypoxia/SD-associated cellular apoptosis was examined by silencing $\beta$-catenin using siRNA. $\beta$-catenin mRNA expression levels were significantly reduced in cells transfected with siRNA- $\beta$-catenin compared with cells transfected with siRNA-NT control (Fig. 3C). Pretreatment with MIF protected MSCs from apoptosis induced by hypoxia/SD (Fig. 4A and B), and significantly decreased the activities of caspases $3 / 7$ and caspase 8 (Fig. 4C and D). However, these effects were abolished by silencing $\beta$-catenin and not by transfection with siRNA-NT (Fig. 4).

MIF enhances MSC survival via inhibition of oxidative stress. Oxidative stress is associated with cellular apoptosis (27), thus, the feedback loop between oxidative stress and the lincRNA-p21-Wnt/ $\beta$-catenin signaling pathway modulated by MIF was explored. Mitochondrial transmembrane potential, ROS generation, SOD activation and lipid peroxidation were examined (Fig. 5). Hypoxia/SD significantly decreased the mitochondrial transmembrane potential (Fig. 5A) and SOD activation (Fig. 5C), while increasing ROS generation (Fig. 5B) and MDA activation (Fig. 5D). Pretreatment with MIF significantly increased the mitochondrial transmembrane potential and SOD activation, and decreased ROS generation of ROS and MDA activation. These anti-oxidant effects of MIF were abolished by ectopic expression of lincRNA-p21 or by silencing $\beta$-catenin (Fig. 5A-D).

\section{Discussion}

MSCs are immune-protected and secrete a wide variety of growth factors that protect the heart from ischemic injury (28). The regenerative potency of these cells has thus been investigated in numerous preclinical and clinical studies, and MSC transplantation in the heart is considered to be a safe therapeutic option in patients with myocardial injury $(29,30)$. However, numerous transplanted cells undergo apoptosis, which is considered to be one of the primary barriers limiting the effectiveness of cell therapy (31). In the present study, hypoxia/SD culture conditions were used to mimic the ischemic environment in vitro, in order to determine whether the survival and potency of transplanted MSCs increase following treatment with MIF.

Plasma MIF levels are increased in patients with myocardial infarction (32), and ischemia also increases MIF expression in rat hearts (33). Excessive MIF reduces cardiac contractility in isolated, perfused rat hearts (34), and has also been reported to enhance the survival of cultured cardiomyocytes (35). The present study revealed that MIF treatment significantly reduced apoptosis in hypoxia/SD-cultured MSCs in vitro, and this reduction was accompanied by inhibition of caspase 3/7 and caspase 8 activities, which are known to be involved in the cellular apoptosis process.

Mammalian genomes encode $>10,000$ lncRNAs, which are RNA molecules $>200$ nucleotides that do not appear to encode proteins (36). IncRNAs have emerged as important regulators
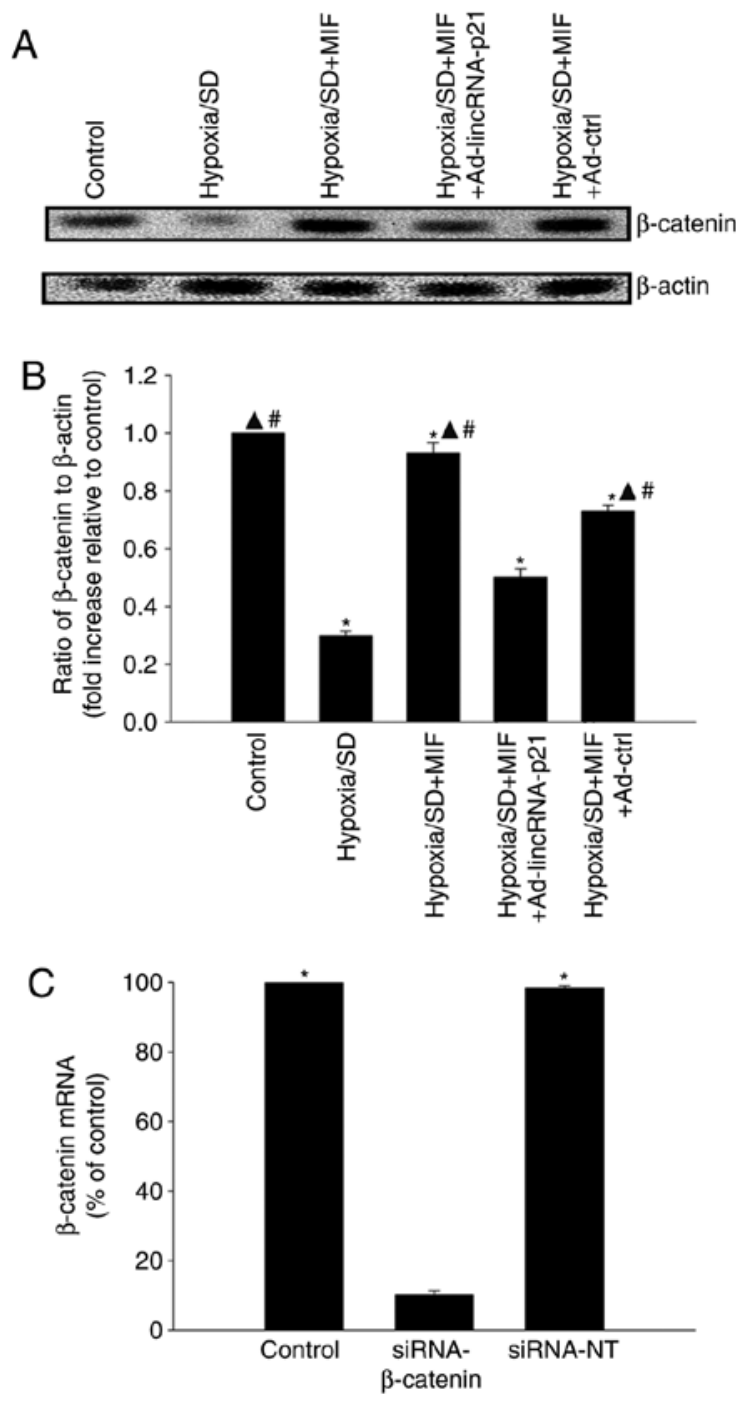

Figure 3. MIF modulated lincRNA-p21-Wnt/ $\beta$-catenin signaling pathway. MSCs were transfected with Ad-lincRNA-p21 or Ad-ctrl followed by hypoxia/SD for $24 \mathrm{~h}$. In parallel experiments, cells were treated with MIF $(100 \mathrm{ng} / \mathrm{ml})$ prior to hypoxia/SD. Untreated MSCs were used as a control. (A) Representative western blot and (B) quantification of $\beta$-actin and $\beta$-catenin in these groups. Each column represents the mean $\pm \mathrm{SD}$ of three independent experiments; ${ }^{*} \mathrm{P}<0.05$ vs. control; ${ }^{\mathbf{A}} \mathrm{P}<0.05$ vs. hypoxia/SD; ${ }^{*} \mathrm{P}<0.05$ vs. hypoxia/SD+MIF+Ad-lincRNA-p21. MSCs were transfected with siRNA against $\beta$-catenin or control siRNA-NT. (C) The siRNA-mediated transfection efficiency was demonstrated by reverse transcription-quantitative polymerase chain reaction. Each column represents the mean \pm standard deviation of three independent experiments; "P<0.05 vs. siRNA- $\beta$-catenin. MIF, macrophage migration inhibitory factor; MSC, mesenchymal stem cells; SD, serum deprivation; siRNA, small interfering RNA; NT, non-targeting; Ad-lincRNA-p21, adenoviral vectors expressing lincRNA-p21; Ad-ctrl, adenoviral vectors expressing control scrambled sequence.

in various cellular processes, including proliferation, survival, autophagy and apoptosis (37). A recent study demonstrated that lncRNAs regulate cell proliferation, apoptosis and autophagy in response to energy stress (38). lincRNA-p21 is a p53-dependent transcriptional target gene with roles in cell cycle arrest and apoptosis in response to DNA damage, and has been revealed to activate damage signaling and cell cycle arrest (39). MIF inhibited p53-dependent death signaling in keratinocytes and exerted a protective effect against cellular apoptosis (17). The results of this study demonstrated that 
A

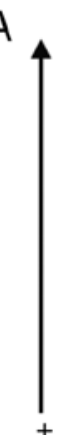

$\frac{t}{\mathrm{~L}}$
Control

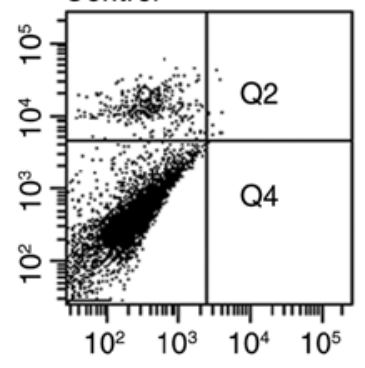

Hypoxia/SD

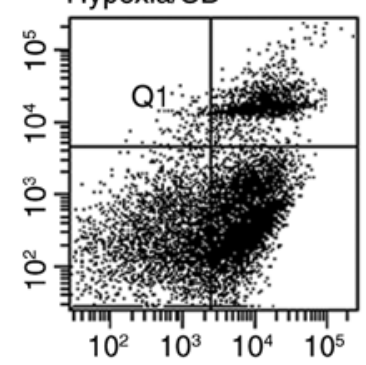

Hypoxia/SD+MIF

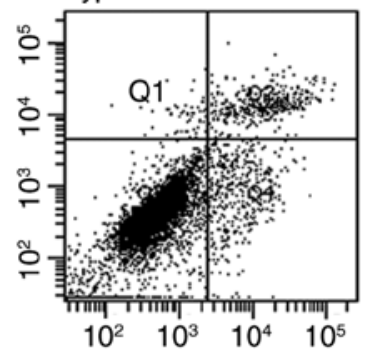

Hypoxia/SD+MIF
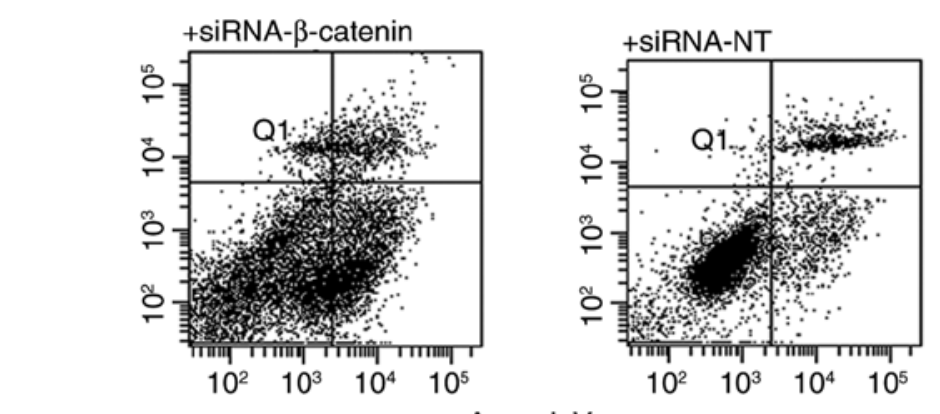

AnnexinV+

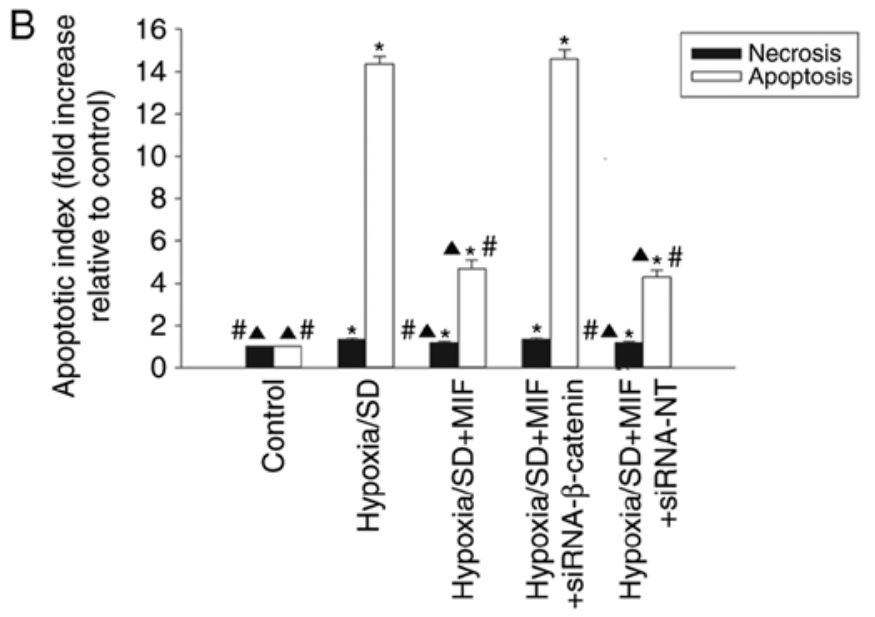

C

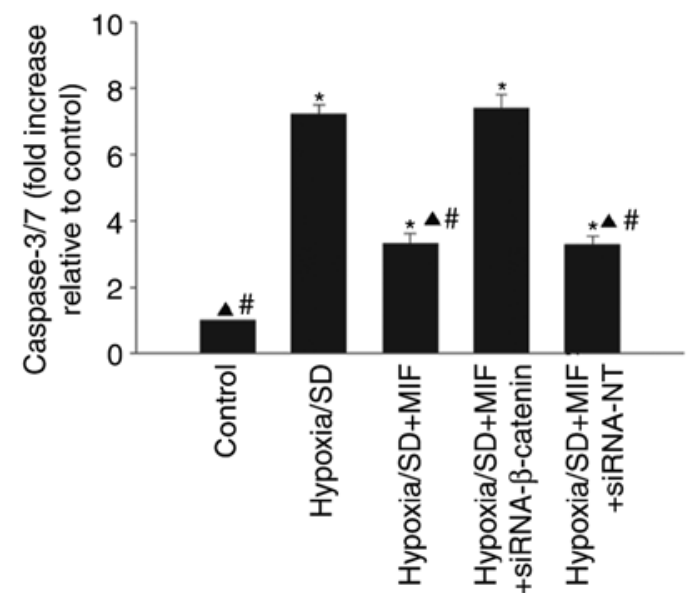

$\mathrm{D}$

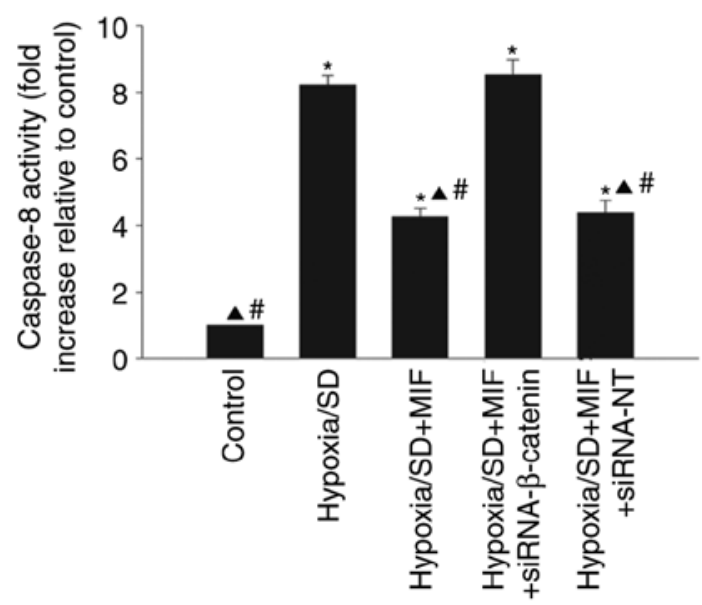

Figure 4. MIF restored the lincRNA-p21-Wnt/ $\beta$-catenin signaling pathway altered by hypoxia/SD induction. MSCs were transfected with siRNA against $\beta$-catenin or control siRNA-NT and incubated under hypoxic/SD conditions for $24 \mathrm{~h}$. In parallel experiments, cells were treated with MIF (100 ng/ml) in the incubation medium throughout the hypoxia/SD treatment period. (A) Representative flow cytometric dot plots of apoptotic cells following Annexin V/propidium iodide staining and (B) quantification of apoptosis. (C) Activities of caspases 3/7 and (D) caspase 8 in cell lysates were measured by ELISA. Each column represents the mean \pm standard deviation of three independent experiments; ${ }^{*} \mathrm{P}<0.05$ vs. control; ${ }^{\Delta} \mathrm{P}<0.05$ vs. hypoxia/SD; ${ }^{*} \mathrm{P}<0.05$ vs. hypoxia/SD+MIF+siRNA- $\beta$-catenin. MIF, macrophage migration inhibitory factor; MSC, mesenchymal stem cells; SD, serum deprivation; siRNA, small interfering RNA; Linc, long intergenic noncoding; NT, non-targeting. 
A
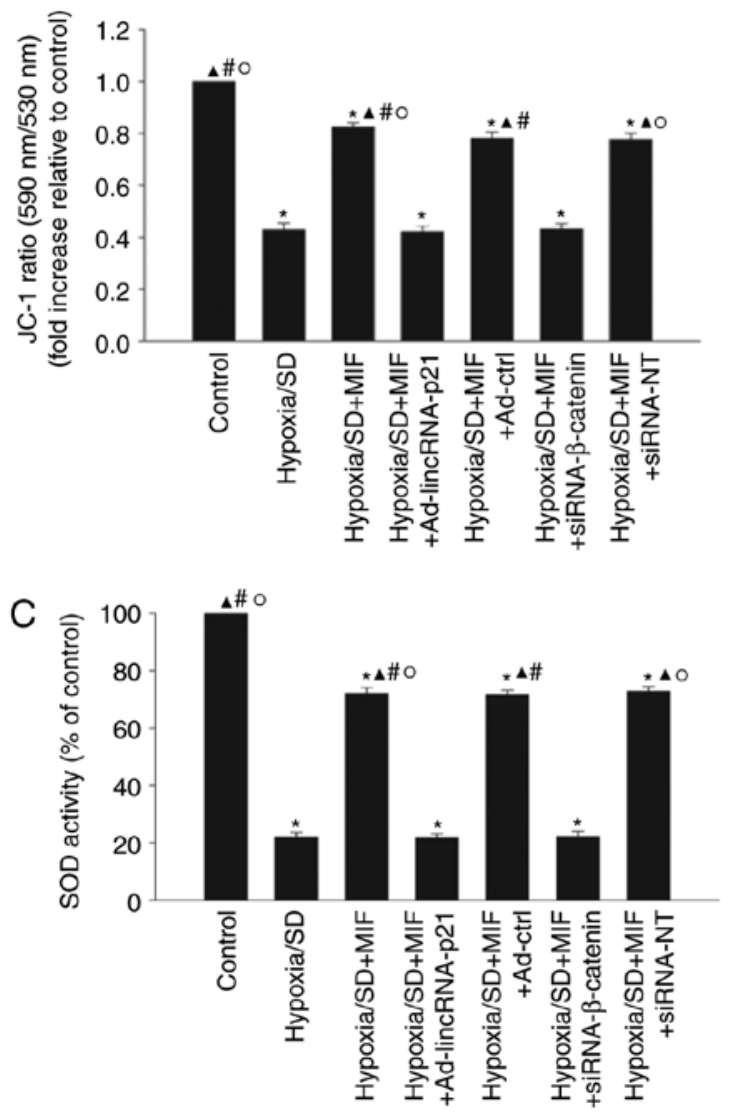

B
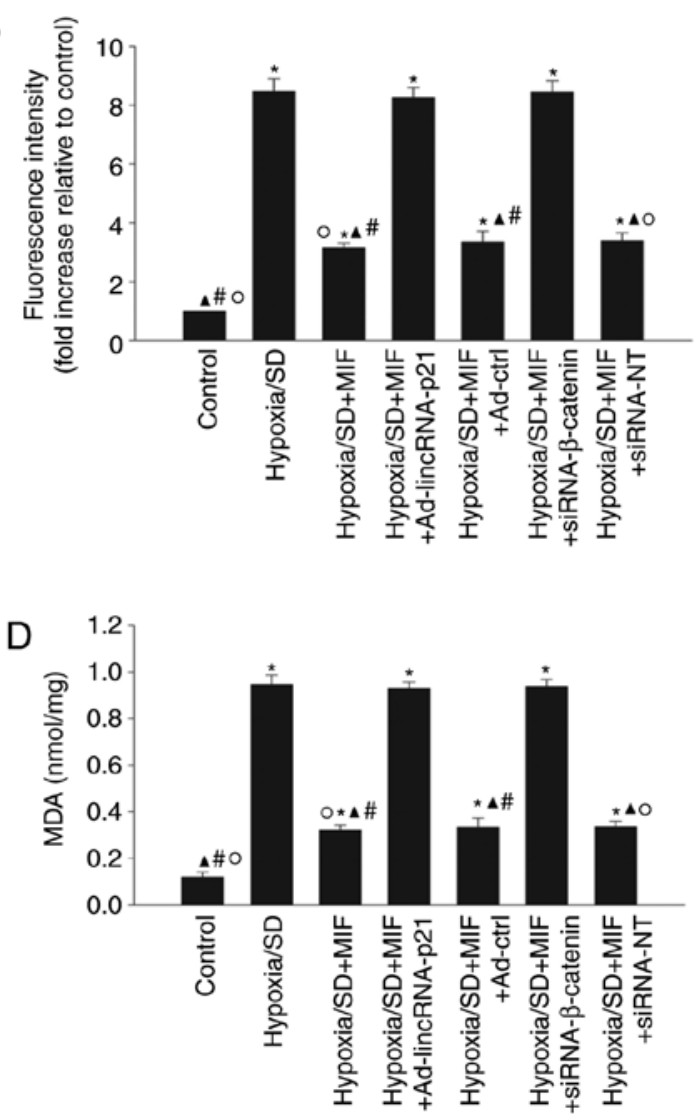

Figure 5. MIF enhanced MSC survival via inhibition of oxidative stress. MSCs were incubated under hypoxic/SD conditions for $24 \mathrm{~h}$. In parallel experiments, cells were transfected with Ad-lincRNA-p21, Ad-ctrl, siRNA- $\beta$-catenin, or siRNA-NT before exposure to hypoxia/SD, and then treated with MIF. MIF (100 ng/ml) was added at the beginning of exposure to hypoxia/SD. Untreated MSCs were used as a control. (A) Mitochondrial membrane potential was measured using JC-1 stain. (B) Intracellular reactive oxygen species production was analyzed by fluorescence spectrophotometry. (C) Superoxide dismutase activity was evaluated by colorimetric assay. (D) Lipid peroxidation was evaluated by malondialdehyde formation. Data represent mean \pm standard deviation from three independent experiments; " $\mathrm{P}<0.05$ vs. control, ${ }^{\wedge} \mathrm{P}<0.05$ vs. hypoxia/SD; ${ }^{\#} \mathrm{P}<0.05$ vs. hypoxia/SD+MIF+Ad-lincRNA-p21; ${ }^{\circ} \mathrm{P}<0.05$ vs. hypoxia/SD+MIF+siRNA- $\beta$-catenin. MIF, macrophage migration inhibitory factor; MSC, mesenchymal stem cells; SD, serum deprivation; siRNA, small interfering RNA; linc, long intergenic noncoding; NT, non-targeting; Ad-lincRNA-p21, adenoviral vectors expressing lincRNA-p21; Ad-ctrl, adenoviral vectors expressing control scrambled sequence.

hypoxia/SD induced apoptosis in MSCs, accompanied by induction of lincRNA-p21, while MIF decreased lincRNA-p21 expression and protected MSCs from injury induced by hypoxia/SD. Overexpression of lincRNA-p21 abolished the anti-apoptotic effect of MIF, confirming that this anti-apoptotic effect was due to inhibition of lincRNA-p21 by MIF.

Wnt/ $\beta$-catenin is a stress signaling kinase pathway and key regulator of energy-generating and consuming pathways. It serves as an essential sensor of cellular energy status and is activated under energy stress conditions, to protect cells against hypoxic injury and death (18). The Wnt/ $\beta$-catenin signaling pathway also regulates organelle-compartmentalized protein folding, and is relevant in the cross-talk between mitochondria and endoplasmic reticulum, thus protecting against oxidative stress (40). $\beta$-Catenin is an important target gene of lincRNA-p21, and lincRNA-p21was demonstrated to inhibit $\beta$-catenin signaling, thereby attenuating the viability, self-renewal and glycolysis of cancer stem cells in vitro (41). Furthermore, MIF enhances the proliferation of neural stem/progenitor cells and promotes neuronal differentiation by activating the Wnt/ $\beta$-catenin signal pathway (42). The current results suggest that the $\mathrm{Wnt} / \beta$-catenin signaling pathway was inhibited by hypoxia/SD and reactivated by exogenous MIF, and this restoration was abolished by lincRNA-p21 overexpression. The relevance of MIF regulation of $\beta$-catenin in hypoxia/SD-induced apoptosis was confirmed by silencing $\beta$-catenin, which attenuated the anti-apoptotic effect of MIF.

Excess oxidative stress causes cell death in the heart (43), and excessive oxidative stress below physiological levels may not only induce myocardial injury in response to ischemia/reperfusion, but may also cause apoptosis of transplanted stem cells (44). IncRNAs are involved in cellular oxidative stress, and recent research revealed that the lncRNAFOXD3-AS1was involved in hypoxia/ROS-induced injury (45). lincRNA-p21was also demonstrated to inhibit HepG2 cell growth by activating endoplasmic reticulum stress (14). A recent study revealed that lincRNA-p21 participated in cellular senescence in MSCs through inducing the oxidative process (16). In addition, MIF-knockout exacerbated doxorubicin-induced mortality and cardiomyopathy, accompanied by cellular apoptosis and ROS generation (46). The results of the present study demonstrated that hypoxia/SD-induced oxidative stress was accompanied by decreased mitochondrial transmembrane potential and activation of SOD, and increased generation of ROS and MDA activation, and these effects were reversed by MIF treatment. The anti-oxidant effects of MIF 
were in turn abolished by overexpression of lincRNA-p21 or silencing of $\beta$-catenin.

In conclusion, the results of this study suggest that MIF acts as an anti-apoptotic factor to counteract hypoxia/SD-induced apoptosis, via a mechanism involving rebalancing the lincRNA-p21-Wnt/ $\beta$-catenin signaling pathway and decreasing oxidative stress.

\section{Acknowledgements}

Not applicable.

\section{Funding}

The present study was supported by the National Natural Science Foundation of China (grant nos. 81600278 to WX and 81500261 to $\mathrm{MH}$ ) and the Medical Science and Technology Project of Zhejiang Province (grant no. 2018K Y517 to $\mathrm{MH}$ ).

\section{Availability of data and materials}

All data generated or analyzed during the present study are included in the published article.

\section{Authors' contributions}

WX and LZ made substantial contributions to the acquisition of data, analysis and interpretation of data; and $\mathrm{MH}$ was involved in conception and design, drafting the manuscript and revising it critically for important intellectual content.

\section{Ethics approval and consent to participate}

All animal procedures were approved by the Institutional Animal Care and Use Committee of Wenzhou Medical University.

\section{Patient consent for publication}

Not applicable.

\section{Competing interests}

The authors declare that they have no competing interests.

\section{References}

1. Chareonthaitawee P, Gersh BJ, Araoz PA and Gibbons RJ: Revascularization in severe left ventricular dysfunction: The role of viability testing. J Am Coll Cardiol 46: 567-574, 2005.

2. Karantalis V, Suncion-Loescher VY, Bagno L, Golpanian S, Wolf A, Sanina C, Premer C, Kanelidis AJ, McCall F, Wang B, et al: Synergistic Effects of combined cell therapy for chronic ischemic cardiomyopathy. J Am Coll Cardiol 66: 1990-1999, 2015.

3. Natsumeda M, Florea V, Rieger AC, Tompkins BA, Banerjee MN, Golpanian S, Fritsch J, Landin AM, Kashikar ND, Karantalis V, et al: A combination of allogeneic stem cells promotes cardiac regeneration. J Am Coll Cardiol 70: 2504-2515, 2017.

4. Abdelwahid E, Kalvelyte A, Stulpinas A, de Carvalho KA Guarita-Souza LC and Foldes G: Stem cell death and survival in heart regeneration and repair. Apoptosis 21: 252-268, 2016.
5. Golpanian S, Wolf A, Hatzistergos KE and Hare JM: Rebuilding the damaged heart: Mesenchymal stem cells, cell-based therapy, and engineered heart tissue. Physiol Rev 96: 1127-1168, 2016.

6. Luedike P, Hendgen-Cotta UB, Sobierajski J, Totzeck M, Reeh M, Dewor M, Lue H, Krisp C, Wolters D, Kelm M, et al: Cardioprotection through S-nitros(yl)ation of macrophage migration inhibitory factor. Circulation 125: 1880-1889, 2012.

7. Calandra $\mathrm{T}$ and Roger T: Macrophage migration inhibitory factor: A regulator of innate immunity. Nat Rev Immunol 3: 791-800, 2003.

8. Qi D, Hu X, Wu X, Merk M, Leng L, Bucala R and Young LH: Cardiac macrophage migration inhibitory factor inhibits JNK pathway activation and injury during ischemia/reperfusion. J Clin Invest 119: 3807-3816, 2009.

9. Zernecke A, Bernhagen J and Weber C: Macrophage migration inhibitory factor in cardiovascular disease. Circulation 117: 1594-1602, 2008.

10. Sun Q, Hao Q and Prasanth KV: Nuclear long noncoding RNAs: Key regulators of gene expression. Trends Genet: S0168-9525(17)30207-X, 2017.

11. Liu C, Yang Z, Wu J, Zhang L, Lee S, Shin DJ, Tran M and Wang L: Long noncoding RNA H19 interacts with polypyrimidine tract-binding protein 1 to reprogram hepatic lipid homeostasis. Hepatology 67: 1768-1783, 2018.

12. Chew CL, Conos SA, Unal B and Tergaonkar V: Noncoding RNAs: Master regulators of inflammatory signaling. Trends Mol Med 24: 66-84, 2018.

13. Bao X, Wu H, Zhu X, Guo X, Hutchins AP, Luo Z, Song H, Chen Y, Lai K, Yin M, et al: The p53-induced lincRNA-p21 derails somatic cell reprogramming by sustaining $\mathrm{H} 3 \mathrm{~K} 9 \mathrm{me} 3$ and CpG methylation at pluripotency gene promoters. Cell Res 25: 80-92, 2015.

14. Yang N, Fu Y, Zhang H, Sima H, Zhu N and Yang G: LincRNA-p21 activates endoplasmic reticulum stress and inhibits hepatocellular carcinoma. Oncotarget 6: 28151-28163, 2015.

15. Yang W, Yu H, Shen Y, Liu Y, Yang Z and Sun T: MiR-146b-5p overexpression attenuates stemness and radioresistance of glioma stem cells by targeting HuR/lincRNA-p21/ $\beta$-catenin pathway. Oncotarget 7: 41505-41526, 2016.

16. Xia W, Zhuang L, Deng $X$ and Hou M: Long noncoding RNA-p21 modulates cellular senescence via the Wnt/ $\beta$-catenin signaling pathway in mesenchymal stem cells. Mol Med Rep 16: 7039-7047, 2017.

17. Yoshihisa Y, Rehman MU, Kondo T and Shimizu T: Role of macrophage migration inhibitory factor in heat-induced apoptosis in keratinocytes. Faseb J 30: 3870-3877, 2016.

18. Gammons $\mathbf{M}$ and Bienz $\mathrm{M}$ : Multiprotein complexes governing Wnt signal transduction. Curr Opin Cell Biol 51: 42-49, 2018

19. Yoon JH, Abdelmohsen K, Srikantan S, Yang X, Martindale JL, De S, Huarte $M$, Zhan $M$, Becker $K G$ and Gorospe $M$ : LincRNA-p21 suppresses target mRNA translation. Mol Cell 47: 648-655, 2012.

20. Yu F, Guo Y, Chen B, Shi L, Dong P, Zhou M and Zheng J: LincRNA-p21 inhibits the Wnt/ $\beta$-catenin pathway in activated hepatic stellate cells via sponging MicroRNA-17-5p. Cell Physiol Biochem 41: 1970-1980, 2017.

21. Zhu W, Chen J, Cong X, Hu S and Chen X: Hypoxia and serum deprivation-induced apoptosis in mesenchymal stem cells. Stem Cells 24: 416-425, 2006.

22. Gore Y, Starlets D, Maharshak N, Becker-Herman S, Kaneyuki U, Leng L, Bucala $\mathrm{R}$ and Shachar I: Macrophage migration inhibitory factor induces $\mathrm{B}$ cell survival by activation of a CD74-CD44 receptor complex. J Biol Chem 283: 2784-2792, 2008.

23. Liu Y, Xiong Y, Xing F, Gao H, Wang X, He L, Ren C, Liu L, So KF and Xiao J: Precise regulation of miR-210 is critical for the cellular homeostasis maintenance and transplantation efficacy enhancement of mesenchymal stem cells in acute liver failure therapy. Cell Transplant 26: 805-820, 2017.

24. Livak KJ and Schmittgen TD: Analysis of relative gene expression data using real-time quantitative PCR and the 2(-Delta Delta C(T)) method. Methods 25: 402-408, 2001.

25. Xia W, Xie C, Jiang M and Hou M: Improved survival of mesenchymal stem cells by macrophage migration inhibitory factor. Mol Cell Biochem 404: 11-24, 2015.

26. Shen Y, Liu Y, Sun T and Yang W: LincRNA-p21 knockdown enhances radiosensitivity of hypoxic tumor cells by reducing autophagy through HIF-1/Akt/mTOR/P70S6K pathway. Exp Cell Res 358: 188-198, 2017. 
27. Maj T, Wang W, Crespo J, Zhang H, Wang W, Wei S, Zhao L, Vatan L, Shao I, Szeliga W, et al: Oxidative stress controls regulatory $\mathrm{T}$ cell apoptosis and suppressor activity and PD-L1-blockade resistance in tumor. Nat Immunol 18: 1332-1341, 2017.

28. Nakamura Y, Wang X, Xu C, Asakura A, Yoshiyama M, From AH and Zhang J: Xenotransplantation of long-term-cultured swine bone marrow-derived mesenchymal stem cells. Stem Cells 25: 612-620, 2007.

29. Amado LC, Schuleri KH, Saliaris AP, Boyle AJ, Helm R, Oskouei B, Centola M, Eneboe V, Young R, Lima JA, et al: Multimodality noninvasive imaging demonstrates in vivo cardiac regeneration after mesenchymal stem cell therapy. J Am Coll Cardiol 48: 2116-2124, 2006

30. Hare JM, Traverse JH, Henry TD, Dib N, Strumpf RK, Schulman SP, Gerstenblith G, DeMaria AN, Denktas AE, Gammon RS, et al: A randomized, double-blind, placebo-controlled, dose-escalation study of intravenous adult human mesenchymal stem cells (prochymal) after acute myocardial infarction. J Am Coll Cardiol 54: 2277-2286, 2009.

31. Ye L, Zhang P, Duval S, Su L, Xiong Q and Zhang J: Thymosin $\beta 4$ increases the potency of transplanted mesenchymal stem cells for myocardial repair. Circulation 128 (11 Suppl 1): S32-S41, 2013.

32. Takahashi M, Nishihira J, Katsuki T, Kobayashi E, Ikeda U and Shimada K: Elevation of plasma levels of macrophage migration inhibitory factor in patients with acute myocardial infarction. Am J Cardiol 89: 248-249, 2002.

33. Yu CM, Lai KW, Chen YX, Huang XR and Lan HY: Expression of macrophage migration inhibitory factor in acute ischemic myocardial injury. J Histochem Cytochem 51: 625-631, 2003.

34. Chagnon F, Metz CN, Bucala R and Lesur O: Endotoxin-induced myocardial dysfunction: Effects of macrophage migration inhibitory factor neutralization. Circ Res 96: 1095-1102, 2005.

35. Wang J, Tong C, Yan X, Yeung E, Gandavadi S, Hare AA, Du X, Chen Y, Xiong $\mathrm{H}$, Ma C, et al: Limiting cardiac ischemic injury by pharmacological augmentation of macrophage migration inhibitory factor-AMP-activated protein kinase signal transduction. Circulation 128: 225-236, 2013.

36. Ulitsky I and Bartel DP: lincRNAs: Genomics, evolution, and mechanisms. Cell 154: 26-46, 2013.
37. Schmitt AM, Garcia JT, Hung T, Flynn RA, Shen Y, Qu K, Payumo AY, Peres-da-Silva A, Broz DK, Baum R, et al: An inducible long noncoding RNA amplifies DNA damage signaling. Nat Genet 48: 1370-1376, 2016.

38. Liu X, Xiao ZD, Zhang J, Lee SW, Wang W, Lee H, Zhuang L, Chen $J$ and Lin HK: LncRNA NBR2 engages a metabolic checkpoint by regulating AMPK under energy stress. Nat Cell Biol 18: 431-442, 2016.

39. Chen S, Liang H, Yang H, Zhou K, Xu L, Liu J, Lai B, Song L, Luo H, Peng J, et al: LincRNa-p21: Function and mechanism in cancer. Med Oncol 34: 98, 2017.

40. Lettini G, Sisinni L, Condelli V, Matassa DS, Simeon V, Maddalena F, Gemei M, Lopes E, Vita G, Del Vecchio L, et al: TRAP1 regulates stemness through Wnt/ $\beta$-catenin pathway in human colorectal carcinoma. Cell Death Differ 23: 1792-1803, 2016.

41. Wang J, Lei ZJ, Guo Y, Wang T, Qin ZY, Xiao HL, Fan LL, Chen DF, Bian XW, Liu J and Wang B: miRNA-regulated delivery of lincRNA-p21 suppresses $\beta$-catenin signaling and tumorigenicity of colorectal cancer stem cells. Oncotarget 6: 37852-37870, 2015.

42. Zhang X, Chen L, Wang Y, Ding Y, Peng Z, Duan L, Ju G, Ren Y and Wang X: Macrophage migration inhibitory factor promotes proliferation and neuronal differentiation of neural stem/precursor cells through Wnt/ $\beta$-catenin signal pathway. Int J Biol Sci 9: 1108-1120, 2013.

43. Matsushima S, Kuroda J, Zhai P, Liu T, Ikeda S, Nagarajan N, Oka S, Yokota T, Kinugawa S, Hsu CP, et al: Tyrosine kinase FYN negatively regulates NOX4 in cardiac remodeling. J Clin Invest 126: 3403-3416, 2016.

44. Han YS, Lee JH, Jung JS, Noh H, Baek MJ, Ryu JM, Yoon YM, Han HJ and Lee SH: Fucoidan protects mesenchymal stem cells against oxidative stress and enhances vascular regeneration in a murine hindlimb ischemia model. Int J Cardiol 198: 187-195, 2015.

45. Zhang D, Lee H, Haspel JA and Jin Y: Long noncoding RNA FOXD3-AS1 regulates oxidative stress-induced apoptosis via sponging microRNA-150. FASEB J 31: 4472-4481, 2017.

46. Xu X, Bucala R and Ren J: Macrophage migration inhibitory factor deficiency augments doxorubicin-induced cardiomyopathy. J Am Heart Assoc 2: e000439, 2013. 